\title{
Contribución de estudios históricos de las escuelas de enfermería a la memoria y la identidad profesional: una revisión integradora
}

\section{Contribution of historical studies about nursing schools to memory and identity of the profession: an integrative review}

\section{Contribuição de estudos históricos sobre escolas de enfermagem para a memória e identidade da profissão: uma revisão integrativa}

Laís de Miranda Crispim Costa; Tầnia Cristina Franco Santos²; Regina Maria dos Santos³;

Maria José Coelho ${ }^{4}$; Isaura Setenta Porto ${ }^{5}$

${ }^{1}$ Enfermeira, Mestre em Enfermagem; Professora Assistente da Escola de Enfermagem e Farmácia-ESENFAR da Universidade Federal de Alagoas-UFAL; Doutoranda do Programa de Pós-Graduação e Pesquisa da Escola da Enfermagem Anna Nery da Universidade Federal do Rio de Janeiro-UFRJ. Endereço: Rua Dr. Roland Simons, no 575/301, Jatiuca. Maceió - Alagoas. CEP: 57.035-552. Brasil. E-mail: laismcc@gmail.com

${ }^{2}$ Professora Associada do Departamento de Enfermagem Fundamental / Escola de Enfermagem Anna Nery / Universidade Federal do Rio de Janeiro. Líder do grupo de pesquisa "História da Enfermagem nas instituições brasileiras do século XX". Bolsista de Produtividade do CNPq.

${ }^{3}$ Enfermeira, Doutora em Enfermagem pela Escola de Enfermagem Anna Nery da Universidade Federal do Rio de Janeiro (EEAN/UFRJ); Docente Permanente do Programa de Pós-Graduação em Enfermagem da ESENFAR/UFAL; Líder do Grupo de Estudos Dona Isabel Macintyre.

${ }^{4}$ Professora da Universidade Federal do Rio de Janeiro, Membro do Conselho Nacional de Desenvolvimento Científico e Tecnológico, do Ministério da Educação/Instituto Nacional de Estudos e Pesquisas Educacional e Ministério da Saúde.

${ }^{5}$ Professora Adjunta da Escola de Enfermagem Anna Nery / UFRJ. Bolsista de Produtividade em Pesquisa do Ministério da Ciência e Tecnologia.

Cómo citar este artículo en edición digital: Crispim Costa, LM., Franco Santos, T.C.,dos Santos, R.Mª., Coelho, Mª.J., \& Setenta Porto, I. (2016). Contribución de estudios históricos de las escuelas de enfermería a la memoria y la identidad profesional: una revisión integradora. Cultura de los Cuidados (Edición digital), 20( 46).

Disponible en: < http://dx.doi.org/10.14198/cuid.2016.46.10>

Correspondencia: Laís de Miranda Crispim Costa. Rua Dr. Roland Simons, No 575, Jatiúca, Maceió- AL, CEP:57035-552. Tel: +55 (82) 93260522

Correo electrónico: laismcc@gmail.com

Recibido: 08/01/2016; Aceptado: 24/07/2016

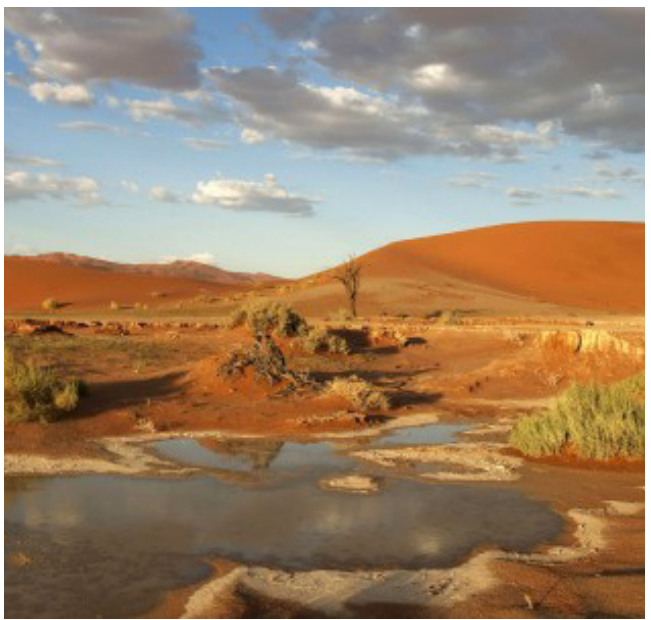

\section{ABSTRACT}

This study aimed to identify and analyze the scientific production of historical studies on nursing schools and their contribution to the memory and identity of the profession. The methodology used was an integrative review. The search was conducted in the databases SciELO, LILACS, MEDLINE and CINAHL. Inclusion criteria were: articles written in Portuguese, English, French or Spanish; be in accordance with the guiding question; and have been published in the last 10 years. The editorials, letters to the reader, biographies and ac- 
counts of experience were excluded. The survey identified 26 articles of sources composing the study sample. The results showed a peak of publication between years 2008 and 2010; the Journal of Nursing in Anna Nery School main periodic disclosure; and Brazil with a significant literature on the subject. Some strengths of the analysis of the articles may be mentioned regarding the contribution to the identity and memory of the profession: a rigid training with religious character; a submission to the medical profession as well as a strong resistance to it; the fight for better positions in the healthcare field; emphasis on professional competence; and the perpetuation of rituals and symbols. All historical studies have proved important for development of the nursing profession as well as to preserve their identity and memory.

Key words: history of nursing, nursing schools, research.

\section{RESUMEN}

Este estudio tuvo como objetivo identificar y analizar la producción científica de los estudios históricos sobre las escuelas de enfermería y su contribución a la memoria y la identidad de la profesión. La metodología utilizada fue la revisión integradora. La búsqueda se realizó en la base de datos SciELO, LILACS, MEDLINE y CINAHL. Los criterios de inclusión fueron: artículos escritos en portugués, inglés, francés o español; estar de acuerdo con la pregunta orientadora; y se han publicado en los últimos 10 años. Se excluyeron editoriales, cartas al lector, biografías y relatos de experiência. El estudio identificó 26 artículos de fuentes que componen la muestra del estudio. Los resultados mostraron un pico de publicación entre los años 2008 y 2010; la Revista de Enfermería de Escuela Anna Nery como el vehículo principal de publicación; y Brasil con una amplia literatu- ra sobre el tema. Algunas fortalezas del análisis de los artículos se pueden mencionar en cuanto a la contribución a la identidad y la memoria de la profesión: una formación rígida de carácter religioso; una sumisión a la profesión médica, así como una fuerte resistencia a la misma; la lucha por mejores posiciones en el campo de la salud; énfasis en la competencia profesional; y la perpetuación de los ritos y símbolos. Todos los estudios históricos han demostrado ser importantes para el desarrollo de la profesión de enfermería, así como a preservar su identidad y la memoria.

Palabras clave: historia de la enfermería, escuelas de enfermería, la investigación.

\section{RESUMO}

Este estudo teve como objetivo identificar e analisar a produção científica de estudos históricos sobre as escolas de enfermagem e sua contribuição para a memória e identidade da profissão. A metodologia utilizada foi uma revisão integrativa. A busca foi realizada nas bases de dados SCIELO, LILACS, MEDLINE e CINAHL. Os critérios de inclusão foram: artigos escritos em português, inglês, francês ou espanhol; estarem de acordo com a questão norteadora; e, terem sido publicados nos últimos 10 anos. Foram excluídos aqueles que se tratavam de editoriais, cartas ao leitor, biografias e relatos de experiência. O levantamento das fontes identificou 26 artigos, compondo a amostra do estudo. Os resultados evidenciaram um pico de publicação entres os anos 2008 e 2010; a Revista de Enfermagem da Escola Anna Nery como principal periódico de divulgação; e, o Brasil com uma expressiva produção sobre a temática. Alguns pontos fortes da análise dos artigos podem ser mencionados no que tange a contribuição para a identidade e memória da 
profissão: uma rígida formação com caráter religioso; uma submissão à categoria médica, assim como uma forte resistência a mesma; a luta por melhores posições no campo da saúde; a ênfase na competência profissional; e, a perpetuação de rituais e símbolos. Todos os estudos históricos se revelaram importantes para desenvolvimento da profissão enfermagem bem como para a preservação da sua identidade e memória.

Palavras-chave: história da enfermagem, escolas de enfermagem, pesquisa.

\section{INTRODUÇÃO}

$O$ conhecimento acerca da enfermagem contribui sobremaneira para compreender o contexto desta profissão bem como para construção de sua memória e identidade. Um dos caminhos para alcançar tais resultados é a produção de conhecimento através de pesquisas sobre sua história, nesse sentido, durante o curso de pós-graduação stricto-senso, na modalidade doutorado, surgiu a necessidade de escrever este artigo, tendo em vista o projeto de tese intitulado: "O Curso de Graduação em Enfermagem da Universidade Federal de Alagoas (1973 - 1979): lutas simbólicas para implantação e consolidação".

Outrossim, a enfermagem se interessa por estudos de sua história, pois o enfermeiro que se apropria desta disciplina, de certo modo de sua própria história, adquire maior domínio e sabedoria para conhecer e discutir de forma crítica, madura e reflexiva aspectos relativos a sua profissão, inclusive desenvolvendo condições de analisar as situações postas na realidade atual (Padilha, Borenstein, 2006). Uma das formas da enfermagem produzir conhecimento na linha de pesquisa da história é através da realização de estudos sobre a criação, implantação e desenvolvimento das Escolas de Enfermagem.

Partindo destas considerações, o presente artigo trata de uma revisão integrativa de literatura, uma técnica de pesquisa que reúne e sintetiza o conhecimento produzido, por meio da análise dos resultados evidenciados nos estudos de muitos autores especializados. Este método de pesquisa indica o conhecimento atual sobre uma temática específica, já que é conduzida de modo a identificar, analisar e sintetizar resultados de estudos independentes sobre o mesmo assunto.

O desenvolvimento da revisão integrativa prevê seis etapas, a saber: seleção de hipóteses ou questões para a revisão; seleção das pesquisas que irão compor a amostra; definição das características das pesquisas; análise dos achados; interpretação dos resultados e, relato da revisão (Souza, Silva, Carvalho, 2010). Diante do exposto, o objetivo deste estudo foi identificar e analisar a produção científica de estudos históricos sobre as escolas de enfermagem e sua contribuição para a memória e identidade da profissão.

\section{METODOLOGIA}

Para guiar a revisão integrativa, formulou-se a seguinte questão norteadora: Qual a contribuição dos estudos históricos sobre as escolas de enfermagem para a construção da memória e identidade da profissão? Para a busca dos artigos foram utilizados apenas dois descritores: 'história da enfermagem' e 'escolas de enfermagem'. A busca foi feita nos seguintes bancos de dados: SCIELO (ScientificElectronic Library Online), LILACS (Literatura Latino-Americana e do Caribe em Ciências da Saúde), MEDLINE (Literatura Internacional em Ciências da Saúde/Medical Literature Analysis and Retrieval System Online) e CI- 
NAHL (Cumulative Index to Nursing \& Allied Health Literature), sendo que nestes dois últimos a consulta foi realizada no laboratório de informática da Escola de Enfermagem Anna Nery da Universidade Federal do Rio de janeiro, instituição licenciada para acesso ao textos completos. A coleta destes dados ocorreu no mês de junho 2014.

A busca foi totalmente realizada pelo acesso on-line e resultou em 232 publicações, sendo que várias publicações se repetiam em mais de um banco de dados. Seguindo a etapa de seleção dos artigos para composição da amostra, procedeu-se a leitura de todos os títulos, seguida da leitura de todos os resumos e quando necessário da leitura do todo o texto no sentido de atender aos critérios de inclusão estabelecidos, quais sejam: artigos escritos em português, inglês, francês ou espanhol; estarem de acordo com a questão norteadora; serem; e, terem sido publicados nos últimos 10 anos. Este último justifica-se por não se tratar de pesquisas voltadas para a prática da enfermagem baseada em evidências. Foram excluídos aqueles que se tratavam de editoriais, cartas ao leitor, biografias e relatos de experiência.

Com base nestes requisitos 26 artigos constituíram a amostra do estudo. Para a análise e posterior síntese dos artigos, foi elaborado um instrumento, contemplando as seguintes variáveis: ano de publicação, título, identificação dos autores, periódico de publicação, volume, número, páginas, objetivos do estudo, resultados e conclusões. A apresentação dos dados e discussão dos resultados obtidos foi organizada em duas etapas, a primeira se refere a apresentação dos dados quantitativos com apoio de um quadro e a segunda está voltada para responder a questão norteadora, realizada de forma descritiva.

\section{APRESENTAÇÃO E DISCUSSÃO DOS RE- SULTADOS}

Será apresentado um panorama geral desses estudos que se referem ao desenvolvimento histórico das escolas de enfermagem no Brasil e no mundo. Segue um quadro (1) sinóptico, que foi montado no sentido de dá visibilidade às Escolas de Enfermagem analisadas nos artigos e suas respectivas localizações (país de origem):

\section{Quadro1: Apresentação dos artigos incluídos na revisão integrativa, segundo ano de publicação, título, escola de enfermagem e localização, Rio de Janeiro, 2014.}

\begin{tabular}{|c|l|l|c|}
\hline ANO & TíTULO & \multicolumn{1}{|c|}{ PAÍS } & $\begin{array}{c}\text { NOME DA ESCOLA } \\
\text { Nursing }\end{array}$ \\
\hline 2004 & Organizational Lifecycle in a School of Nursing & $\begin{array}{l}\text { Estados } \\
\text { Unidos }\end{array}$ & $\begin{array}{c}\text { DePauw University School of } \\
\text { Beraldo }\end{array}$ \\
\hline 2004 & $\begin{array}{l}\text { Criação e implantação da escola de enfermagem } \\
\text { Hermantina Beraldo, Gestão Celina Viegas. }\end{array}$ & Brasil & $\begin{array}{c}\text { Escola de Enfermagem Hermantina } \\
\text { Evolving as a University-Wide School of Nursing }\end{array}$ \\
\hline 2005 & $\begin{array}{l}\text { Eeunião de Diretoras de Escolas de Enfermagem: um } \\
\text { Cenário de Lutas Simbólicas no Campo da Educação } \\
\text { em Enfermagem (1943-1945) }\end{array}$ & $\begin{array}{l}\text { Brasil } \\
\text { University School of Nursing }\end{array}$ \\
\hline 2005 & $\begin{array}{l}\text { La Escuela Nacional Superior de Enfermeras de } \\
\text { Colombia. }\end{array}$ & Colômbia & $\begin{array}{c}\text { Escuel Nacional Superior de } \\
\text { Enfermeras da Universidade } \\
\text { Nacional de Colômbia }\end{array}$ \\
\hline
\end{tabular}




\begin{tabular}{|c|c|c|c|}
\hline 2006 & $\begin{array}{l}\text { As Circunstâncias do Processo de Reconfiguração da } \\
\text { Escola Profissional de Assistência a Psicopatas do } \\
\text { Distrito Federal. }\end{array}$ & Brasil & $\begin{array}{l}\text { Escola Profissional de Enfermeiros } \\
\text { e Enfermeiras do Serviço de } \\
\text { Assistência a Psicopatas do Distrito } \\
\text { Federal }\end{array}$ \\
\hline 2006 & $\begin{array}{l}\text { Aspectos da Formação Profissional na Escola de } \\
\text { Enfermagem Alfredo Pinto (1943-1949). }\end{array}$ & Brasil & $\begin{array}{l}\text { Escola de Enfermagem Alfredo } \\
\text { Pinto }\end{array}$ \\
\hline 2006 & $\begin{array}{l}\text { Circunstâncias Histórico-Sociais de criação da primeira } \\
\text { escola de enfermagem no Vale do Paraíba - "Dom } \\
\text { Epaminondas". }\end{array}$ & Brasil & $\begin{array}{l}\text { Escola de enfermagem Dom } \\
\text { Epaminondas }\end{array}$ \\
\hline 2007 & $\begin{array}{l}\text { O jogo de forças na reorganização da escola } \\
\text { Profissional de Enfermeiros e Enfermeiras. }\end{array}$ & Brasil & $\begin{array}{l}\text { Escola Profissional de Enfermeiros } \\
\text { e Enfermeiras }\end{array}$ \\
\hline 2008 & $\begin{array}{l}\text { Perfil de uma escola de enfermagem do Sul do Brasil } \\
\text { no período das fundadoras. }\end{array}$ & Brasil & $\begin{array}{c}\text { Escola de Enfermagem da } \\
\text { Universidade Federal do Rio } \\
\text { Grande do Sul }\end{array}$ \\
\hline 2008 & $\begin{array}{l}\text { Criação e consolidação do Curso de Enfermagem na } \\
\text { Universidade de Brasília: uma história de tutela (1975- } \\
\text { 1986). }\end{array}$ & Brasil & $\begin{array}{l}\text { Curso de Enfermagem na } \\
\text { Universidade de Brasília }\end{array}$ \\
\hline 2008 & $\begin{array}{l}\text { Resistência à Liderança Norte-Americana na Formação } \\
\text { da Enfermeira Brasileira (1934-1938). }\end{array}$ & Brasil & Escola de Enfermagem Anna Nery \\
\hline 2008 & $\begin{array}{l}\text { Looking to the future: Early twentieth-century school } \\
\text { nursing in Queensland. }\end{array}$ & Austrália & Nursing school of Queensland \\
\hline 2008 & $\begin{array}{l}\text { Emblemas e Rituais: Reconstruindo a História da } \\
\text { Escola de Enfermagem Hermantina Beraldo. }\end{array}$ & Brasil & $\begin{array}{l}\text { Escola de Enfermagem Hermantina } \\
\text { Beraldo }\end{array}$ \\
\hline 2008 & $\begin{array}{l}\text { O Cotidiano dos Alunos na Escola de Enfermagem } \\
\text { Alfredo Pinto (1949-1956). }\end{array}$ & Brasil & $\begin{array}{l}\text { Escola de Enfermagem Alfredo } \\
\text { Pinto }\end{array}$ \\
\hline 2009 & $\begin{array}{l}\text { Perfil dos Homens Formados na Escola de Enfermagem } \\
\text { Da Universidade De São Paulo (1950-1990). }\end{array}$ & Brasil & $\begin{array}{l}\text { Escola de Enfermagem da } \\
\text { Universidade de São Paulo }\end{array}$ \\
\hline 2009 & $\begin{array}{l}\text { The University of Minnesota School of Nursing: } \\
\text { A Generation Ahead }\end{array}$ & $\begin{array}{l}\text { Estados } \\
\text { Unidos }\end{array}$ & $\begin{array}{l}\text { Escola de Enfermagem da } \\
\text { Universidade de Minnesota }\end{array}$ \\
\hline 2009 & $\begin{array}{l}\text { A Escola de Enfermagem do Hospital São Paulo e seu } \\
\text { primeiro currículo (1939-1942). }\end{array}$ & Brasil & $\begin{array}{l}\text { Escola de Enfermagem do Hospital } \\
\text { São Paulo }\end{array}$ \\
\hline 2010 & A Inserção de Waleska Paixão Na Enfermagem. & Brasil & $\begin{array}{l}\text { Escola de Enfermagem Carlos } \\
\text { Chagas }\end{array}$ \\
\hline 2010 & $\begin{array}{l}\text { Memória de Egressos da Escola de Enfermagem da } \\
\text { Universidade De São Paulo. }\end{array}$ & Brasil & $\begin{array}{c}\text { Escola de Enfermagem } \\
\text { da Universidade de São Paulo }\end{array}$ \\
\hline 2010 & $\begin{array}{l}\text { A road not taken: the proposal for } \\
\text { a Harvard School of Nursing }\end{array}$ & $\begin{array}{l}\text { Estados } \\
\text { Unidos }\end{array}$ & Harvard School of Nursing \\
\hline 2010 & $\begin{array}{l}\text { Escolas e Cursos de Enfermagem na História da } \\
\text { Profissão no Brasil (1890-1922). }\end{array}$ & Brasil & $\begin{array}{l}\text { Escolas e cursos de enfermagem no } \\
\text { recorte temporal de 1890-1922. }\end{array}$ \\
\hline 2011 & $\begin{array}{l}\text { Los inicios de la profesionalización de la enfermería en } \\
\text { Colombia. }\end{array}$ & Colômbia & $\begin{array}{l}\text { Escuela de enfermería de } \\
\text { Colombia }\end{array}$ \\
\hline
\end{tabular}




\begin{tabular}{|c|c|c|c|}
\hline 2011 & $\begin{array}{l}\text { Development of the School of Nursing, Midwifery, } \\
\text { and Public Health at Siriraj, Thailand 1896-1971: } \\
\text { A historical study. }\end{array}$ & Tailândia & $\begin{array}{l}\text { Escola de Enfermagem, obstetrícia, } \\
\text { e Saúde Pública da Siriraj. }\end{array}$ \\
\hline 2012 & $\begin{array}{l}\text { Primeira Década do Curso de Enfermagem na } \\
\text { Universidade Federal De Juiz de Fora: } 1979-1989 .\end{array}$ & Brasil & $\begin{array}{l}\text { Curso de Graduação em } \\
\text { Enfermagem da Universidade } \\
\text { Federal de Juiz de Fora. }\end{array}$ \\
\hline 2013 & $\begin{array}{l}\text { Writing History: Case Study of the University } \\
\text { of Victoria School of Nursing }\end{array}$ & Canadá & $\begin{array}{c}\text { University } \\
\text { of Victoria School of Nursing }\end{array}$ \\
\hline
\end{tabular}

Foram preservados os títulos e os periódicos de acordo com o idioma de publicação para não correr o risco de comprometer o entendimento após a tradução. Pode-se perceber que, de acordo o critério inclusão do ano de publicação, houve publicações em todos os anos que compuseram a amostra, ou seja, de 2004 a 2013, pois o ano da coleta de dados não foi considerado. Podemos ver que em uma década a maioria das produções destas pesquisas históricas envolvendo o desenvolvimento de escolas de enfermagem situa-se no centro deste período, ou seja, entre os anos e 2008 e 2010 é que se concentram metade desta produção, exatos $50 \%$.

Analisando-se o veículo de publicação desses trabalhos, $14(53,8 \%)$ são artigos publicados em periódicos brasileiros e 12 (46,2\%) em periódicos estrangeiros, demonstrando um relativo equilíbrio quando comparando estas duas categorias. Pelo quadro 1 observa-se uma certa pulverização das publicações, prevalecendo o número de 1 artigo por cada periódico, tanto nos estrangeiros como nos nacionais. Sendo que um dado chama bastante atenção, a existência de duas revistas que concentram a maioria das publicações, uma estrangeira (Cultura de los Cuidados) e uma brasileira (Revista de Enfermagem da Escola Anna Nery).

Na Revista Cultura de los Cuidados foram publicados 4 artigos (15,3\%) desta revisão, dos quais 3 se referem a estudos produzidos no Brasil, ou seja, este periódico se configura como um importante veículo de divulgação dos estudos sobre a história da enfermagem brasileira. Vale enfatizar que este periódico destina-se a contribuir para a divulgação de estudos sobre o desenvolvimento do conhecimento histórico, antropológico, fenomenológico e teórico da enfermagem.

$\mathrm{O}$ achado mais relevante se refere a Revista de Enfermagem da Escola Anna Nery, representando mais de um quarto das produções (27\%). Este resultado bastante expressivo provavelmente tem ligação com o funcionamento nesta Instituição do Núcleo de Pesquisa em História da Enfermagem Brasileira (Nuphebras), criado em 08 de dezembro de 1933, cuja principal finalidade é promover o desenvolvimento da pesquisa em História da Enfermagem no Brasil, congregando pesquisadores da própria escola e de outras instituições.

Vemos também dois outros periódicos brasileiros que apresentaram 2 artigos (7,6\%) cada um, a Revista Brasileira de Enfermagem - REBEn e a Revista Latino-americana de Enfermagem. A primeira tem a missão divulgar a produção científica, de diferentes áreas do saber, que seja do interesse da enfermagem, incluindo a que expresse o projeto político da Associação Brasileira de Enfermagem ABEn. É importante considerar que a REBEn é uma revista independente, não vinculada a 
nenhum programa de pós-graduação e recebe produções sem nenhuma restrição, desde que respeitem as suas normas, abrangendo portanto pesquisa de cunho histórico sobre a profissão.

Já a segunda, a Revista Latino-americana de Enfermagem teve sua primeira edição publicada em 1993, ou seja, com mais de duas décadas de existência já se configura na atualidade como um periódico nacional de circulação internacional, cujo foco é publicar resultados de pesquisas de enfermagem que contribuam para o avanço do conhecimento científico e para prática profissional, com impacto significativo (Qualis A) para a área da enfermagem.

Em relação ao país de realização dos estudos, houve um resultado expressivo de estudos realizados em instituições brasileiras de ensino de enfermagem. Dos trabalhos avaliados, 17 (65,3\%) são estudos realizados no Brasil. Certamente este dado tem relação com duas das bases de dados consultadas. SciELO, que é uma biblioteca eletrônica que abrange uma coleção selecionada de periódicos científicos brasileiros, e LILACS, uma base Latino-Americana de informação bibliográfica em ciências da saúde. Por outro lado, este dado pode ser analisado também como um aspecto positivo, pois evidencia a produção de autores brasileiros na região da América Latina e Caribe, contribuindo para visibilidade da história da enfermagem brasileira.

Em relação ao objetivo desta revisão, qual seja, identificar e analisar a produção científica de estudos históricos sobre as escolas de enfermagem e sua contribuição para a memória e identidade da profissão, observamos nos artigos que compõem a amostra que todos apresentam aspectos que contribuem para esta memória e identidade, alguns de maneira mais tímida e outros de forma mais contundente.
Cabe esclarecer a diferença entre identidade individual e coletiva. "É possível definir identidade de uma pessoa como algo individual, com um conjunto de caracteres próprios e exclusivos como: nome, filiação, local e data de nascimento, profissão, sexo, e principalmente se pensada através das impressões digitais marcas que caracterizam a identidade de uma pessoa. A identidade coletiva seria o conjunto de características pelas quais algo é definitivamente reconhecível ou conhecido. A identidade profissional seria um tipo de identidade coletiva, com base em traços ou características comuns a todos que exercem determinada atividade" (Campos, Oguisso, 2008).

Nesse sentido, toma-se como entendimento que a identidade profissional não deve ser avaliada a partir de imposições e sim de um contexto social onde é (re)produzida e que na busca desta identidade não é tarefa fácil delimitar uma profissão. Assim, a presente revisão não pretende traçar um caminho cronológico de construção desta identidade e sim discutir aspectos que se sobressaíram na amostra do estudo.

Destaca-se em algumas escolas "o disciplinamento e a regulação do modo de vida de seus agentes com o emprego de dispositivos que delimitavam o lugar que cada indivíduo deveria ocupar no espaço" (Fertig, Xavier, Souza, 2008). Tomando como exemplo o regime interno da primeira escola do estado de São Paulo, a Escola de Enfermeiras do Hospital de São Paulo - EEHSP implantada em 1939, as alunas eram supervisionadas 24 horas por dia pelas professoras religiosas (Silva, Gallian, 2009). E também a Escola da Enfermagem da Universidade de Minnesota, criada em março de 1909, que enfatiza a sua influência no pensamento de seus estudantes por mais de cem anos (Disch, 2009). 
Outras escolas estudadas na amostra deste estudo também relataram a existência do regime de internato em alguma fase do seu desenvolvimento, sobretudo na época de criação, como é o caso da Escola de Enfermagem Carlos Chagas - EECC e a Escola de Enfermagem de Greencastle da Universidade de Pauw (Santos, Caldeira, Moreira, 2010; Porter, Bean, 2004). Já na Escola de Enfermagem Hermantina Beraldo - EEHB o controle da pontualidade e assiduidade se fazia mediante uma 'cardeneta vermelha' e na Escola Nacional de Enfermeiras da Colômbia as alunas eram obrigadas a estagiar nos hospitais aos domingos e feriados, evidenciando mais uma vez uma rígida formação (Araújo, Nascimento, Caldeira, 2004; Mora, 2009; Carvajal, 2011; Toledo, Santos, Araújo, Almeida Filho, 2008).

Estudos com recorte temporal mais recente, como é o caso da primeira década de funcionamento do Curso de Enfermagem da Universidade de Juiz de Fora (1979-1989), também trazem resquícios do controle exercido pelos docentes sobre os discentes (Jesus, Santos, Figueiredo, Gava, Pereira, 2012). Com as mudanças na legislação da educação brasileira e a instituição do sistema de créditos nas universidades, o estudante passa a ter direito de planejar sua vida acadêmica e os docentes mostraram dificuldade de aceitar esta nova realidade.

E mesmo com este sistema ainda persistiam ferramentas de controle, como a avaliação rigorosa do estudante em relação ao seu desempenho individual e coletivo (Jesus, Santos, Figueiredo, Gava, Pereira, 2012). Enfim, várias escolas, com ênfase naquelas criadas ainda na segunda metade do século XIX e primeira metade do século XX, tinham uma formação moral rígida, com excessivo controle, jogos de poder e um grau de exigência bastante elevado para as alunas (Meirelles, Amorim, 2008).

Este controle tinha início antes mesmo do ingresso nas escolas, de modo que vários atributos eram requeridos das possíveis ingressantes, como provas escritas sobre conhecimentos diversos (português, matemática, história, geografia), vários tipos de exames de saúde, atestado de sanidade mental, cartão de vacina (Araújo, Nascimento, Caldeira, 2004). Para a Escola de Enfermagem da Universidade de Indiana (Indianapolis - Estados Unidos) houve uma fase que exigia também uma boa reputação (Mcbride, Yeager, Farley, 2005). Já a Escola de Enfermagem Alfredo Pinto na década de 1940 requeria a apresentação de um atestado de bons costumes (Bess, Amorim, 2006).

Durante a reconfiguração (primeira metade do século XX) da Escola Profissional de Assistência a Psicopatas do Distrito Federal é possível elencar mais de quarenta qualidades desejáveis para enfermeira, "entre as quais boa memória, exatidão, prontidão de espírito, doçura, capacidade de observação, previdência, paciência ilimitada, sangue frio de domínio absoluto de si mesma" (Amorim, Barreira, 2006).

Algumas destas qualidades nos fazem perceber que a religião ocupa um lugar privilegiado na história da enfermagem, a ponto ter escola que ministrava disciplina sobre religião católica (Araújo, Nascimento, Caldeira, 2004; Toledo, Santos, Araújo, Almeida Filho, 2008). A Escola de Enfermagem Anna Nery - EEAN também sofreu uma influência religiosa muito forte, sobretudo na gestão de Laís Netto dos Reis (Almeida Filho, Santos, Baptista, Lourenço, 2005). Igualmente a Escola Nacional Superior de Enfermeiras da Colômbia a partir de sua primeira diretora, Helen Howitt (Mora, 2009).

A Escola de Enfermagem de Greencastle da Universidade de Pauw declara que um dos 
seus objetivos era conservar e desenvolver a saúde física, o caráter moral e a vida religiosa de seus alunos. Por outro lado, esta mesma escola se preocupava com a excelência intelectual e criativa dos estudantes, o que demonstra uma preocupação com o desenvolvimento de enfermeiros competentes (Porter, Bean, 2004).

Sobre este aspecto, a Escola de Enfermagem da Universidade de Indiana apresentou uma rápida expansão da graduação em enfermagem em termos de qualidade de formação, contribuindo também para visibilidade da profissão (Mcbride, Yeager, Farley, 2005). O Currículo da Escola Nacional Superior de Enfermeiras da Colômbia também se preocupou em desenvolver as mais variadas competências para os seus egressos, incluindo orientação sanitária, estágio rural, conteúdos de fisioterapia e nutrição (profissão ainda não existentes no campo da saúde), um diferencial a época - primeira metade do século XX (Mora, 2009).

Há que se enfatizar, quando se aborda o avanço tecnológico e desenvolvimento de competências na formação, que a Escola de Enfermagem de Harvard, criada ainda no século XIX, apresenta na sua proposta curricular conteúdos voltados para o cuidado domiciliar profissional ou "home care", situação que ressalta o avanço desta escola no que tange a formação do enfermeiro no mundo (Ward, 2010). Uma outra escola também estrangeira (Escola de Enfermagem da Universidade de Victoria Canadá) traz outros aspectos bem avançados da sua proposta curricular, com diferentes metodologias de ensino, integração da aprendizagem afetiva, iniciativa de consolidar habilidades psicomotoras, integração teoria e prática e diminuição das fronteiras hierárquicas entre professores e alunos (Scaia, Young, 2013).

Historicamente o avanço, e a consequente visibilidade, da enfermagem no campo da saúde revelaram embates com uma categoria hegemônica no mundo, a medicina. O que não foi diferente nesta revisão. Mesmo em escolas de instituições renomadas, a exemplo da Escola de Enfermagem de Harvard à época de sua criação, havia uma preocupação de que a responsabilidade da formação de enfermeiras deveria ser dos médicos do país, de modo que fosse mantida uma situação de submissão, aos moldes da enfermagem pré-nightingaleana(Scaia, Young, 2013).

$\mathrm{O}$ artigo que trata desta escola traz como fonte primária um livro didático para enfermeiros, escrito por um médico obviamente, datado de 1885, que define o papel do enfermeiro da seguinte forma: "para o médico, o primeiro dever é a obediência, fidelidade absoluta às suas ordens, mesmo que a necessidade das medidas prescritivas não lhe seja compreensível” (Scaia, Young, 2013). As primeiras iniciativas de organização do ensino de Enfermagem em Queensland na Austrália também demonstraram que houve uma resistência da categoria médica, que almejava uma categoria profissional subserviente as suas ordens, como ressalta o artigo uma "enfermeira auxiliar". Aspecto que foi revertido não somente pela atuação das enfermeiras, mas pelo contexto social, incluindo os efeitos de duas guerras mundiais que determinou a inserção do enfermeiro no campo da saúde (Madsen, 2008).

Também a primeira fase de desenvolvimento da primeira escola de Enfermagem da Tailândia contou com a forte influência da medicina, com um modelo de formação de aprendiz de enfermagem ensinado por médicos do sexo masculino (Thaweeboon, Peachpansri, Pochanapan, Senachack, Pinyopasakul, 2011).

No Brasil o modelo clássico da enfermagem moderna, que foi implantado pelas enfermeiras americanas através da criação da Esco- 
la de Enfermagem do Departamento Nacional de Saúde Pública (atual Escola de Enfermagem Anna Nery), enfatizava a hierarquia, a disciplina e a subordinação à medicina (Santos, Lopes, Porto, Fonte, 2008). Outro achado bastante intrigante é que o processo de criação e consolidação do curso de enfermagem da Universidade de Brasília foi bastante conturbado, pela resistência do curso de medicina, que considerava a criação de um departamento de enfermagem traria uma força política que perturbaria a calma necessária ao regime (Cardoso, Dytz, 2008).

De maneira semelhante, o jogo de forças na reorganização da Escola Profissional de Enfermeiras e Enfermeiros - EPEE (atual Escola de Enfermagem Alfredo Pinto) na década de 1930 no Brasil também evidenciou a ameaça que a enfermagem representava à categoria médica, tanto que os psiquiatras desta instituição "resistiam às propostas de elevação do capital cultural na formação das alunas e dos alunos da EPEE, já que este desenvolvimento reduziria a distância e a distinção entre médicos e enfermeiras, no campo da psiquiatria, na medida em que estas agentes se tornassem capazes de elaborar um discurso próprio, denunciando as relações de dominação a que eram sujeitas" (Amorim, Barreira, 2007).

Até a imprensa médica desta época, diante das iniciativas de profissionalização da enfermagem, divulgava que o enfermeiro precisava ser um auxiliar do médico, capaz de compreender suas prescrições para executá-la conscientemente e com responsabilidade, pois os hospitais estavam infestados de pessoal desprovido de preparação (Porto, Amorim, 2010). Certamente as cidades que não contavam com escolas de enfermagem tinham um cenário na área da saúde bastante preocupante, como evidenciado na região do Vale do Paraíba onde os serviços e os cuidados de enfermagem ficavam sob responsabilidade de irmãs de caridade treinadas por médicos, antes da implantação da Escola Dom Epaminondas (Antunes, Sanna, 2006).

Diante do exposto, a criação de algumas escolas já se dava na tentativa de modificar esta situação, é o caso da Escola de Enfermagem do Hospital de São Paulo fundada em 1939, primeira instituição de ensino de enfermagem em nível superior na cidade. Esta escola também sofreu influência da Escola Paulista de Medicina, com a proposta de formar o enfermeiro com um perfil baseado na competência, nos valores éticos, morais e espirituais, cujo lema era "não viver, senão para sevir" (Silva, Gallian, 2009).

Outro fator que se relevou importante para discussão de identidade e memória nesta revisão foi a incorporação de rituais e emblemas, fato apenas evidenciado nas pesquisas realizadas em instituições de ensino da enfermagem do Brasil. Essa preocupação de uma escola em perpetuar os ritos simbólicos demonstra a clara de intenção de consagrar um modelo de enfermeira para a sociedade (Meirelles, Amorim, 2008). Na EEAN os rituais e emblemas da profissão foram institucionalizados pelas enfermeiras norte-americanas desde a inauguração da escola (Santos, Lopes, Porto, Fonte, 2008). Na EEHB havia também a passagem da lâmpada (símbolo da enfermagem) durante a cerimônia de colação de grau e o uso de uniformes de galas pelas alunas e professoras (Araújo, Nascimento, Caldeira, 2004; Toledo, Santos, Araújo, Almeida Filho, 2008).

O que está em jogo é a construção de uma identidade e a consagração de um modelo de enfermeira para a sociedade brasileira, nesta direção, "os rituais institucionais têm a função de atribuir e distinguir qualidades novas, con- 
ferindo ao grupo os meios para atuar sobre a realidade social" (Santos, Lopes, Porto, Fonte, 2008).

Todos estes dados deixam claro que estudos históricos sobre o desenvolvimento da profissão enfermagem são importantes para a preservação da memória da profissão. A história da enfermagem desvela as transformações da profissão e nos permite compreender movimentos da construção, desconstrução e reconstrução do passado, na perspectiva da recuperação e da preservação da memória coletiva (Padilha, Borenstein, 2006; Campos, Oguisso, 2008; Toledo, Santos, Araújo, Almeida Filho, 2008; Meirelles, Amorim, 2008; Santos, Lopes, Porto, Fonte, 2008; Mota, Freitas, Agra, Viana, Takashi, Oguisso, 2010).

Sem dúvida a preservação de fontes como os documentos históricos, imagens e registros, é o que permite a construção de versões históricas balizadas e eruditas, bem como a preservação da memória de uma instituição, no presente caso das escolas de enfermagem no Brasil e no mundo, uma vez que "a memória coletiva faz parte das grandes questões das sociedades desenvolvidas e das sociedades em vias de desenvolvimento, das classes dominantes e das classes dominadas, lutando, todas, pelo poder ou pela vida, pela sobrevivência e pela promoção" (Le Goff, 2013).

\section{CONSIDERAÇÕES FINAIS}

Pela caracterização das publicações analisadas, consideramos que os artigos científicos que compuseram a amostra desta revisão tiveram um pico de publicação nos anos 2008, 2009 e 2010. Em relação ao veículo de publicação, esta revisão evidenciou que Revista de Enfermagem da Escola Anna Nery representa o principal periódico de divulgação das pesquisas históricas vinculadas ao desenvolvimento de escolas de graduação em enfermagem. O Brasil também apresentou expressiva produção sobre a temática.

Diante de todo o exposto alguns pontos fortes da análise dos artigos podem ser mencionados no que tange a contribuição para a identidade e memória da profissão: uma rígida formação com caráter religioso; uma submissão à categoria médica, assim como uma forte resistência a mesma; a luta por melhores posições no campo da saúde; a ênfase na competência profissional; e, a perpetuação de rituais e símbolos.

Estes estudos históricos contribuíram para compreender parte da trajetória profissional, além da memória e da identidade da profissão da enfermagem, bem como as representações e significados que têm sido socialmente atribuídos ao enfermeiro e ao que ele faz.

Contudo, todas essas questões são importantes para a escrita da história da enfermagem, estimulando ainda mais um compromisso perene com nossa profissão. Ainda assim, sugere-se que os pesquisadores da área de história da enfermagem reforcem a necessidade de pesquisar sobre suas escolas de origem.

\section{REFERÊNCIAS}

- Almeida Filho, A.J., Santos, T.C.F., Baptista, S.S., y Lourenço, L.H.S.C. (2005). Reunião de Diretoras de Escolas de Enfermagem: um Cenário de Lutas Simbólicas no Campo da Educação em Enfermagem (1943-1945). Texto Contexto Enferm, 14(4), 528-36. Recuperado de http://www.scielo.br/pdf/tce/v14n4/a09v14n4.pdf

- Amorim, W.M., y Barreira, I.A. (2006). As circunstâncias do processo de reconfiguração da escola profissional de assistência a psicopatas do Distrito Federal. Esc. Anna Nery Rev. Enfermagem, 10(2), 195-203. Recuperado de http://www.scielo.br/pdf/ean/v10n2/a05v10n2.pdf

- Amorim, W.M., y Barreira, I.A. (2007). O jogo de forças na reorganização da Escola Profissional de Enfermeiros 
e Enfermeiras. Revista Brasileira de Enfermagem, 60(1), 55-61. Recuperado de http://www.scielo.br/pdf/reben/ v60n1/a10v60n1.pdf

- Antunes, A.P.S. y Sanna, M.C. (2006). Circunstâncias histórico-sociais de criação da primeira escola de enfermagem no Vale do Paraíba - "Dom Epaminondas". Esc. Anna Nery Rev. Enfermagem, 10(1), 54-63. Recuperado de http://www.scielo.br/pdf/ean/v10n1/ v10n1a07.pdf

- Araújo, M.A., Nascimento, E.S., y Caldeira, V.P. (2004). Criação e implantação da escola de enfermagem Hermantina Beraldo - Gestão Celina Viegas. Rev. Mineira de Enfermagem, 8(3), 358-63. Recuperado em http://www.reme.org.br/artigo/detalhes/723

- Bess, M.N., y Amorim, W.M. (2006). Aspectos da Formação Profissional na Escola de Enfermagem Alfredo Pinto (1943-1949). Esc. Anna Nery Rev. Enfermagem, 9 (3), 64-74. Recuperado de http://www.scielo.br/pdf/ean/ v10n1/v10n1a08.pdf

- Campos, P.F.S., y Oguisso, T. (2008). A Escola de Enfermagem da Universidade de São Paulo e a reconfiguração da identidade profissional da enfermagem Brasileira. Rev Bras Enferm, 61(6), 892-8. Recuperado em http://www.scielo.br/pdf/reben/v61n6/ a17v61n6.pdf

- Cardoso, F.A., y Dytz, J.L.G. (2008). Criação e Consolidação do Curso de Enfermagem na Universidade de Brasília: uma história de tutela (1975-1986). Esc. Anna Nery Rev. Enfermagem, 12(2), 251-7. Recuperado de http://www.scielo.br/pdf/ean/v12n2/v12n2a08.pdf

- Carvajal, B.C. (2011). Los Inicios de la Profesionalización de la Enfermería en Colombia. Invest Educ Enferm, 29(2), 269-85. Recuperado em http://www.redalyc.org/ articulo.oa?id=105222400015

- Disch, J. (2009). The University of Minnesota School of Nursing: A Generation Ahead. Creative Nursing, 15(2), 108-10.

- Fertig, A., Xavier, I.H.F., y Souza, L.M. (2008). Perfil de uma Escola de Enfermagem do Sul do Brasil no período das fundadoras. Revista Gaúcha de Enfermagem, 29(1), 98-103. Recuperado em https://www.lume. ufrgs.br/bitstream/handle/10183/23591/000656286. pdf? sequence $=1$

- Jesus, M.C.P., Santos, S.M.R., Figueiredo, M.A.G., Gava, G.F., y Pereira, F.O. (2012). Primeira Década do Curso de Enfermagem na Universidade Federal De Juiz De Fora: 1979 -1989. Esc. Anna Nery Rev. Enfermagem (impr.),16(2), 255- 262.

- Le Goff, J. (2013). Memória e História. Campinas, SP: Editora da Unicamp.

- Madsen, W. (2008). Looking to the future: Early twentieth-century school nursing in Queensland. Contemporary Nurse, 30, 133-141.

- Mcbride, A.B., Yeager, L., y Farley S. (2005). Evolving as a University-Wide School of Nursing. Journal of Professional Nursing, 21(1), 16-22.

- Meirelles, M.R., y Amorim, W.M. (2008). O Cotidiano dos Alunos na Escola de Enfermagem Alfredo Pinto (1949-1956). Rev.Latino-am.Enfermagem, 16(6). Recuperado de http://www.redalyc.org/articulo. oa?id=281421896011

- Mora, A.L.V. (2009). La Escuela Nacional Superior de Enfermeras de Colombia. Cultura de los cuidados, 13(25),36-45. Recuperado em http://rua.ua.es/dspace/ bitstream/10045/11538/3/CC_25_06.pdf

- Mota, N.F., Freitas, G.F., Agra, A.L., Viana, J.M.L., Takashi, M.H., y Oguisso, T. (2010). Memória de Egressos da Escola de Enfermagem da Universidade de São Paulo. Cultura de los Cuidados, 14(28), 31-38.

- Padilha, M.I.C.S., y Borenstein, M.S. (2006). História da Enfermagem: Ensino, Pesquisa e Interdisciplinaridade. Esc Anna Nery R Enferm, 10(3), 532-8. Recuperado em http://www.scielo.br/pdf/ean/v10n3/v10n3a24.pdf

- Porter, M.L., y Bean, J.P. (2004). Organizational Lifecycle in a School of Nursing. Western Journal of Nursing Research, 26(4), 444-60.

- Porto, F., y Amorim, W.M. (2010). Escolas e Cursos de Enfermagem na História da Profissão no Brasil (18901922). Cultura de los Cuidados, 14(27),40-45. Recuperado de http://rua.ua.es/dspace/bitstream/10045/14388/1/ CC 27 05.pdf

- Santos, G.F., Caldeira, V.P., y Moreira, S.A. (2010). A Inserção de Waleska Paixão na Enfermagem. Esc Anna Nery Rev Enferm, 14,(2): 268-274. Recuperado em 
http://www.scielo.br/pdf/ean/v14n2/08.pdf

- Santos, T.C.F, Lopes, G.T.L., Porto, F., y Fonte, A.S. (2008). Resistência à Liderança Norte-Americana na Formação da Enfermeira Brasileira (1934-1938). Rev. Latino-am.Enfermagem, 16(1),130-135. Recuperado de http://www.scielo.br/pdf/rlae/v16n1/pt 19.pdf

- Scaia, M.R., y Young, L. (2013). Writing History: Case Study of the University of Victoria School of Nursing. International Journal of Nursing Education Scholarship, 10(1), 1-8.

- Silva, M.R.G. y Gallian, D.M.C. (2009). A Escola de Enfermagem do Hospital São Paulo e seu primeiro currículo (1939-1942). Rev Bras Enferm, 62(2), 317-22. Recuperado em http://www.scielo.br/pdf/reben/v62n2/ a24v62n2.pdf

- Souza, M.T., Silva, M.D., y Carvalho, R. (2010). Revi- são integrativa: o que é e como fazer. Einstein, 8(1Pt1), 102-6. Recuperado em http://astresmetodologias.com/ material/O_que_e_RIL.pdf

- Thaweeboon, T., Peachpansri, S., Pochanapan, S., Senachack, P., y Pinyopasakul, W. (2011). Development of the School of Nursing, Midwifery, and Public Health at Siriraj, Thailand 1896-1971: A historical study. Nursing and Health Sciences, 13, 440-446.

- Toledo, J.R., Santos, T.C.F., Araújo, M.A., y Almeida Filho, A.F. (2008). Emblemas e Rituais: Reconstruindo a História da Escola de Enfermagem Hermantina Beraldo. Esc. Anna Nery Rev. Enfermagem, 12(2), 243-250. Recuperado de http://www.scielo.br/pdf/ean/v12n2/ v12n2a07.pdf

- Ward, F. (2010). A road not taken: the proposal for a Harvard School of Nursing. Nursing Inquiry, 17(2), 128-141.

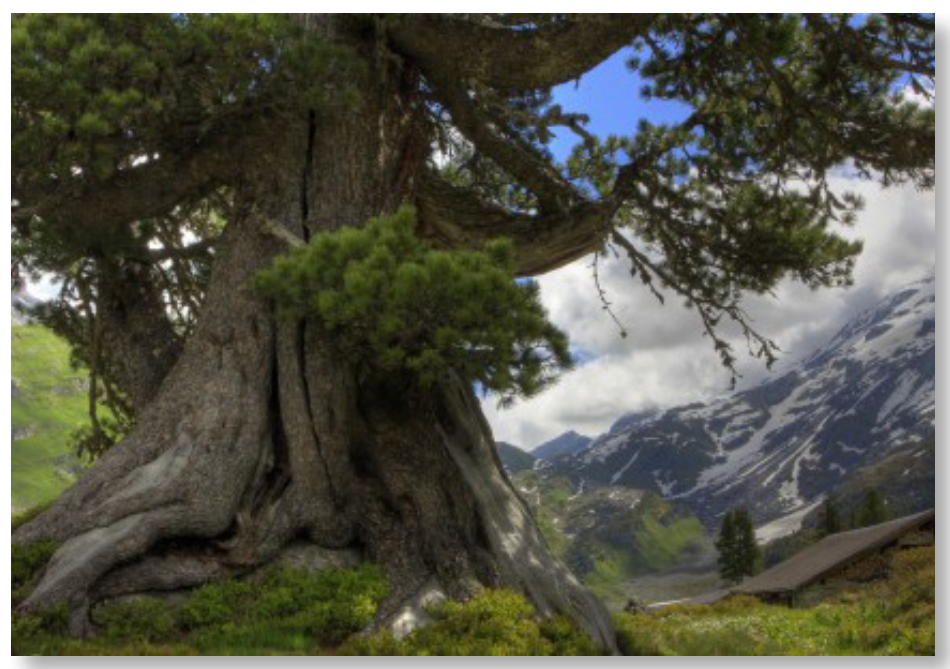

